\title{
The Planarity of the Stickface Motion in the Field Hockey Hit
}

\author{
Alexander P. Willmott ${ }^{1,2}$ and Jesús Dapena ${ }^{1}$ \\ ${ }^{1}$ Department of Kinesiology, Indiana University, Bloomington, IN, USA \\ ${ }^{2}$ Department of Sport, Coaching \& Exercise Science, University of Lincoln, Lincoln, \\ UK
}

Running Title: The planarity of the field hockey hit.

Keywords: Field hockey, hit, kinematics, swing plane 


\begin{abstract}
The field hockey hit is an important but poorly understood stroke. This study investigated the planarity of the stickface motion during the downswing, in order to better characterise the kinematics and to assess the suitability of planar pendulum models for simulating the hit. Thirteen experienced female field hockey players were filmed executing hits with a single approach step, and the kinematics of the centre of the stickface were measured. A method was developed for identifying how far back from impact the stickface motion was planar. Orthogonal regression was used to fit least-squares planes to the stickface path during sections of the downswing of varying length, with each section ending at impact. A section was considered planar if the RMS residual between the stickface path and the fitted plane was less than $0.25 \%$ of the distance traveled by the stickface during that period. On average the stickface motion was planar for the last $83 \pm 12 \%$ of its downswing path, with the length of the planar section ranging from $1.85 \mathrm{~m}$ to $2.70 \mathrm{~m}$. The suitability of a planar model for the stickface motion was supported, but further investigation of the stick and arm kinematics is warranted.
\end{abstract}




\section{Introduction}

The field hockey hit uses a two-handed swinging motion to produce high ball velocity, making it an effective choice for a shot at goal or for a long-distance pass. Despite its importance, however, there have been few quantitative studies of the movement. Brétigny et al. (2008) investigated the kinematics of the upper body in two types of hit, but the only three-dimensional (3D) studies to date that have analysed the kinematics of both the stick and body were those undertaken by Chivers and Elliott (1987) and Elliott and Chivers (1988). All of these studies reported only selected parameters, however, and there has been no investigation of the underlying kinetics.

One aspect of the hit that has been briefly mentioned in the literature, and that has important implications for the modeling of the activity, is the planarity of the motion. In his coaching manual, Wein (1979) reported that the backswing and the subsequent downswing occur in a vertical plane. However, Chivers and Elliott (1987) described the downswing motion as curving "backwards then downwards and forward in an oblique plane”, with the downswing motion not restricted to the same plane as the backswing. These authors did not provide any details of how the planes were defined or oriented, or information concerning how closely the motion of the stick followed the planes.

The concept of a "swing plane" has been much more widely discussed in the golf literature. It was first popularised by Cochran and Stobbs (1968) who, based on qualitative analysis of high-speed film of professional golfers, observed that the clubhead moves in a single inclined plane during "the major part" of the downswing and "much of" the follow-through. Whilst noting that the hands and clubhead actually moved in slightly different planes, Cochran and Stobbs concluded that it 
was an acceptable simplification to model the golf swing as a double pendulum in which the arms and club moved in a single plane during the downswing. The same assumption of planarity has been central to many subsequent pendulum models for golf (such as Jorgensen, 1999, and Sprigings \& MacKenzie, 2002), although its validity has been challenged (see, for example, Coleman \& Anderson, 2007). The concept of the swing plane in golf has recently been reviewed by Kwon and Casebolt (2009).

There have been no studies of the swing plane in field hockey. The objective of this project was to investigate the planarity of the stickface motion during the downswing of the field hockey hit, as a first step in assessing the suitability of a planar model for this activity. In doing this, a new method was developed that allowed more precise localisation of the start of the planar section than has been possible in previous studies that have considered the swing plane in golf. (An earlier version of this approach was described in abbreviated form by the current authors in a 2008 conference proceedings).

\section{Methods}

\section{Participants}

Thirteen experienced female field hockey players, all NCAA Division I varsity players or coaches, participated in the study (height $=1.67 \pm 0.06 \mathrm{~m}$; mass $=63 \pm 6$ kg; mean $\pm s$ ). Institutional Committee for the Protection of Human Subjects approval was obtained for the study and all participants provided informed consent. 


\section{Trial and filming procedures}

Before each trial began, three markers were attached to the stick. These consisted of $19 \mathrm{~mm}$ wide electrical tape wrapped around the proximal shaft (just distal to the hands), the distal shaft, and the stick toe. (See Figure 1.) The locations of the two shaft markers relative to the proximal end of the stick were measured, along with the length of the stick. (See Figure 1.)

Once the marking of the stick was completed, each participant was asked to hit a stationary ball at a goal cage approximately $18 \mathrm{~m}$ away. In order to standardise the task, the participant was permitted to take only one step towards the ball. (See Figure 2.) Unlimited practice hits were permitted until the participant was comfortable with the task, after which three hits were filmed. The hits were filmed with two Locam motion-picture cameras. The cameras were set at a nominal rate of $200 \mathrm{fps}$; the exact rate was subsequently determined from analysis of timing lights recorded on the films. Any hit that missed the target was rejected; the highest speed hit from those remaining was selected for digitisation. For two of the participants only two trials were recorded, due to film constraints. Both hits of both players were on target, and the fastest hit of each player was included in the study.

\section{Acquisition of stick marker coordinates}

Analysis of the stick motion began at the last frame before the left foot lost contact with the ground at the start of the forward step. The two-dimensional (2D) locations of the three stick markers were digitised manually in every frame from this instant until the last frame before the stick-ball impact. Due to the lack of mechanical synchronisation between the cameras, the precise correspondence of frames between the two cameras was established using a procedure based on the methods of 
Pourcelot and colleagues (Pourcelot, Audigié, Degueurce, Denoix, \& Geiger, 1997; Pourcelot, Audigié, Degueurce, Geiger, \& Denoix, 2000) and Yeadon and King (1998). Quintic spline functions developed by Woltring (1986) were then used, with no smoothing, to interpolate between the digitised data and compute the marker locations at $0.005 \mathrm{~s}$ intervals up to $0.005 \mathrm{~s}$ before impact. The spline functions were also used to extrapolate to a provisional set of coordinates for the positions of the markers at the instant of impact.

Separately, the three stick markers were also digitised in every frame from the one immediately after impact through to the last frame in which all of the stick markers were still visible in both cameras. The length of this additional period varied between 17 and 32 frames. Two-dimensional digitised coordinates were obtained at $0.005 \mathrm{~s}$ intervals, starting at $0.005 \mathrm{~s}$ after impact, using the same synchronisation and quintic spline interpolation techniques described above.

Within each of the two periods, the DLT method (Abdel-Aziz \& Karara, 1971; Walton, 1981) was applied to the digitised data to obtain 3D coordinates for the stick markers at each instant. The coordinates were expressed in an inertial reference frame $\mathrm{R}$ with origin at ground level below the centre of the ball in its preimpact position. The reference frame's axes were defined by vectors $\mathbf{X}, \mathbf{Y}$, and $\mathbf{Z}$. $\mathbf{Y}$ was horizontal and pointed towards the goal; $\mathbf{Z}$ pointed vertically upwards; $\mathbf{X}$ was the cross-product of $\mathbf{Y}$ and $\mathbf{Z}$. (See Figure 2.)

Correction of gross digitising errors and estimation of stick marker positions at impact

For each participant, both the pre- and post-impact 3D data for the markers were input to a computer program designed to identify gross errors in the digitising 
process. The time-dependent $\mathrm{X}, \mathrm{Y}$ and $\mathrm{Z}$ coordinates of each marker were viewed separately, and any obvious errors in the coordinates were corrected manually using a graphical interface.

The same graphical interface was used to refine the estimated impact positions that had been obtained by quintic spline extrapolation from the pre-impact data. The latter procedure may produce an excessive amount of random error (Gordon \& Dapena, 2006), and the estimates of locations at impact were improved in this study through a novel approach that took advantage of the additional information contained in the post-impact data. Despite the anticipated abrupt change in velocity during impact, the location curves from the pre- and post-impact phases must converge at the instant of contact. The graphical interface was used to display the provisional estimates of the $\mathrm{X}, \mathrm{Y}$ and $\mathrm{Z}$ coordinates at impact alongside the pre- and post-impact values. Where necessary, the impact position was adjusted to bring it into correspondence with both extrapolation forward from the pre-impact data and extrapolation backward from the post-impact data. The post-impact data were not used further in this study.

\section{Smoothing of the location data for the markers}

The 3D locations for all the markers were then smoothed over the period up to and including the instant of impact, using quintic spline functions (Woltring, 1986). Woltring's Mode 1 was chosen, with the effective cutoff frequency selected on a participant-by-participant basis. These cutoff frequencies ranged from 30 to $42 \mathrm{~Hz}$.

\section{Calculation of locations and velocities for reconstructed stick landmarks}

The smoothed location data for the three stick markers were used to calculate the 3D 
coordinates of two further landmarks. (See Figure 1.) As the first step in this process, three stick axes were defined for each instant of the trial: $\mathbf{X}_{\mathbf{S}}$ (anteroposterior), $\mathbf{Y}_{\mathbf{S}}$ (mediolateral) and $\mathbf{Z}_{\mathbf{S}}$ (longitudinal). $\mathbf{Z}_{\mathbf{S}}$ pointed from the proximal shaft marker to the distal shaft marker. The cross-product of $\mathbf{Z}_{\mathbf{S}}$ with a vector pointing from the stick toe marker to the distal shaft marker yielded $\mathbf{X}_{\mathbf{S}}$. $\mathbf{Y}_{\mathbf{S}}$ was the cross-product of $\mathbf{Z}_{\mathbf{S}}$ and $\mathbf{X}_{\mathbf{S}}$. The location of the heel of the stick was calculated using the location of the proximal shaft marker, the direction of $\mathbf{Z}_{\mathbf{S}}$ and the measurements made directly on the stick. The centre of the stickface was defined as the point halfway across the width of the stick in the centre of the stickhead curve (Figure 1), and its location was determined from the position of the heel of the stick, the directions of the three stick axes, and average offsets along these axes determined from a representative sample of sticks. The centre of the stickface was considered the stick's intended point of impact with the ball, although in reality the contact point would have varied between trials.

Quintic spline functions with no further smoothing were then fitted to the reconstructed position data for the centre of the stickface. The first derivative gave the instantaneous velocity of this point, and its instantaneous speed was calculated from the instantaneous velocity components using Pythagoras’ Theorem.

\section{Definition of the start of the downswing}

Some players executing a field hockey hit use a looped swing in which there is no clear point of reversal that could be used to define the transition from backswing to downswing (See below.) For this activity a more robust definition for the start of the downswing was found to be the instant when the stickface began its final uninterrupted increase in speed through to impact. The gradual nature of the initial 
speed increase presented some practical difficulties for the detection of this instant. It was necessary to prevent small spurious speed increases due to noise in the data from producing a false early detection, but it was also necessary to avoid the use of an excessively conservative criterion that would delay the detection. A satisfactory method was found after some preliminary testing. For each instant of the swing, the average rate of change of speed during the 0.01-second interval that followed it was calculated. The earliest instant after which the average rate of change of stick speed exceeded $2 \mathrm{~m} / \mathrm{s}^{2}$ for all subsequent 0.01 -second intervals up to impact was assumed to represent the start of the speed increase. One participant's stickface speed decreased during the final $0.01 \mathrm{~s}$ before impact; this was not considered to violate the above definition of final speed increase.

The planarity of the motion of the centre of the stickface during the downswing The planarity of the stickface's motion was investigated by fitting least-squares planes to the stickface location data over a number of sections of the downswing. Each of these sections ended at the impact position, but their starting points were spaced at even intervals back along the stickface's downswing path. In order to give equal weight to all parts of the stickface's curved path, the original points (sampled at even time intervals) were first resampled at even distances. Quintic spline interpolation (Woltring, 1986) was used, with no smoothing, to calculate points at $0.05 \mathrm{~m}$ intervals from the position at impact back along the stickface path.

The first section to which a plane was fitted included three points, and comprised the last $0.10 \mathrm{~m}$ of the stickface path. Each subsequent section included a further $0.05 \mathrm{~m}$ along the path. Best-fit planes to the stickface location data were determined using Total Least Squares (Orthogonal) Regression which, unlike 
Multiple Regression, allows for error in all three coordinates (Nievergelt, 1994) and minimises the perpendicular distances between the 3D points and the fitted plane.

For any given section of the stickface path, the centroid $\left(x_{0}, y_{0}, z_{0}\right)$ was determined by averaging the $\mathrm{X}, \mathrm{Y}$ and $\mathrm{Z}$ coordinates of the stickface over that portion of the downswing. A matrix $\mathbf{M}$ was then constructed with the instantaneous coordinates relative to the centroid. The singular vector corresponding to the smallest singular value of matrix $\mathbf{M}$ represented the unit vector normal to the least-squares plane (Nievergelt, 1994). The singular vectors were calculated using subroutine SVD from the EISPACK Fortran library (Grabow, Boyle, Dongarra, \& Moler, 1977).

The direction of the normal vector was used in defining the plane for the section being analysed. The equation for the plane was expressed in the form:

$$
a x+b y+c z+d=0
$$

where $a, b$ and $c$ were the $\mathrm{X}, \mathrm{Y}$ and $\mathrm{Z}$ components, respectively, of the unit vector normal to the plane. The fourth coefficient in the equation for the plane, $d$, was calculated using Equation 1 and the location of the centroid, since the least-squares plane must pass through this point (Nievergelt, 1994; Shakarji, 1998). Rearranging Equation 1 gives the following solution for $d$ :

$$
d=-\left(a x_{0}+b y_{0}+c z_{0}\right)
$$

The orientation of the plane was also described relative to the inertial reference frame using a pair of angles. The first indicated the tilt of the plane relative to horizontal, and was calculated as the angle between the normal vector and the 
vertical $\mathrm{Z}$ axis. The second was a measure of the alignment of the plane with the target direction, and was calculated as the angle between the $\mathrm{Y}$ axis and the line of intersection between the fitted plane and the horizontal XY plane.

The fit of each plane to the data points was estimated quantitatively by examining the perpendicular distances of the individual points from the plane. These residuals were calculated by inputting each point's $\mathrm{X}, \mathrm{Y}$ and $\mathrm{Z}$ coordinates into the left-hand side of Equation 1. For each section of the swing, the Root Mean Square (RMS) of the residuals was determined in metres. However, these values will tend to be smaller for shorter portions of the downswing, which would complicate planarity comparisons between sections of widely differing lengths. The RMS residuals were therefore expressed as a percentage of the distance traveled by the stickface during that section of the downswing. This relative RMS (or RRMS) was used as the measure of the closeness of fit between a given section of the stickface path and the least-squares plane calculated for it.

In order to determine an appropriate cutoff value for RRMS beyond which the stickface motion would no longer be considered planar, a range of values between $0.1 \%$ and $1.0 \%$ of the path length were investigated. A value of $0.25 \%$ of the path length was ultimately selected as the criterion to be used in this study; this choice is explained in the Discussion.

\section{Results}

Backswing shape and downswing length

Preliminary observation of the recorded hits indicated that there was great variety in the shape of the path followed by the stickface during the backswing. For six participants the stickface path was similar during the backswing and downswing. In 
the remaining seven players the stickface moved back along a curved path that was above its path during the subsequent downswing. These two types of swing were classified as "straight” and “looped” respectively, and participants were given identifiers whose first character denoted the type of swing that they used:

Participants S1 to S6 used a straight swing; Participants L1 to L7 used a looped swing. The stickface paths during sample straight and looped swings are shown in Figure 3. The distance that the stickface traveled during the downswing varied between $2.17 \mathrm{~m}$ in Participant S1 and $3.38 \mathrm{~m}$ in Participant L1, and it tended to be longer in the looped swings $(2.88 \pm 0.28 \mathrm{~m})$ than in the straight swings $(2.45 \pm$ $0.28 \mathrm{~m})$

Changes in RRMS with increasing length of the downswing section Figure 4 shows, for selected participants, how RRMS varied with the starting position of the section of the downswing being analysed. Of all 13 players, Participant S2 had the smallest RRMS over the length of the whole downswing (0.10\% of the path length); Participant L1 had the largest (2.85\%).

For all 13 players, RRMS was lower than $0.40 \%$ of the distance traveled by the stickface for at least the final $2.00 \mathrm{~m}$ of the downswing. In the straight swings, the downswing remained close to the fitted plane beyond this point: The highest RRMS over the entire downswing was $0.62 \%$ for Participant S5. In the looped swings, RRMS rose steadily as the distance of the starting point from impact increased past $2.00 \mathrm{~m}$, but the RRMS for the entire downswing remained below $1.50 \%$ in four of the seven looped swing players. It exceeded $2.00 \%$ of the downswing path length only in Participant L1. 


\section{Length and orientation of the planar region}

Figure 5 shows the stickface location at the start of the planar section in selected participants when an RRMS value of $0.25 \%$ was used as the cutoff. The length of the planar section of the downswing was $2.30 \pm 0.26 \mathrm{~m}$ in the straight swings. This section represented $94 \pm 6 \%$ of the downswing length for this type of swing, with values ranging from $85 \%$ for Participant S1 to $100 \%$ for Participant S3, and it accounted for $90 \pm 9 \%$ of the speed added to the stickface during the downswing of these swings. The planar portion was shorter in the looped swings than in the straight swings both in absolute terms $(2.11 \pm 0.14 \mathrm{~m})$ and as a proportion of the downswing path length (74 \pm 8\%). Values for the latter ranged from 63\% for Participant L4 to 86\% for Participant L7. The speed added to the stickface over the planar section accounted for $66 \pm 12 \%$ of the total speed added during the downswing.

The fitted plane was oriented at $43.2 \pm 4.5^{\circ}$ and $38.2 \pm 7.3^{\circ}$ to horizontal in the straight and looped swings, respectively. The line of intersection of the plane with the horizontal plane pointed $6.6 \pm 7.1^{\circ}$ to the left of the target direction in the straight swings and $1.9 \pm 6.2^{\circ}$ to the right of the target direction in the looped swings.

The closeness of fit between the stickface path and the plane

Figure 6 demonstrates the tight correspondence between the stickface path and the fitted plane in the planar region identified using the selected RRMS cutoff of $0.25 \%$ of the path length. The absolute residuals between the stickface and the plane are shown, expressed in metres, for the whole downswing but the planar region starts only at the point designated as ST. The stickface position at the start of the downswing was farthest from the plane in the looped swings, and the stickface path approached the plane from above in all seven swings of this type. 


\section{Discussion}

This investigation has shown that the motion of the stickface is planar for a large portion of the downswing of the field hockey hit. This is especially true for players who use a straight swing. In these hits, the whole downswing may fall within the criterion for planarity. Following a looped backswing, the stickface starts high and its initial motion is primarily downwards before it becomes planar for approximately the last $2 \mathrm{~m}$ of the downswing. The latter pattern seems to fit with Chivers and Elliott's (1987) description of the hit, as given in the Introduction.

The region of the field hockey hit in which the stickface motion is planar appears to be broadly similar to that in which the clubhead motion is planar in golf. Shin, Casebolt, Lambert, Kim and Kwon (2008) found that the latter motion was planar between the instants when the club shaft was horizontal in the downswing and when it was horizontal in the follow-through. Kwon and Casebolt (2009) described this section of the swing as the "impact portion” and suggested that the earlier part of the downswing may not need to be planar as long as it sets the clubhead up for planar motion through this crucial section of the swing. The approach used by Shin et al. (2008) did not preclude the possibility that the planar region in golf may begin earlier than the horizontal position in the downswing; their results indicated only that the clubhead path became planar somewhere between the instants in the downswing at which the club shaft was vertical and horizontal. In the current study, at the start of the planar section the stick shaft was at an average of $35^{\circ}$ above horizontal in the straight swings, and $20^{\circ}$ above horizontal in the looped swings.

The precise start of the planar region is, however, dependent upon the choice of the criterion for planarity. The use in this study of the relative measure RRMS rather than an absolute one has been explained above. The selection of an RRMS 
cutoff value was based upon the overall closeness of fit between the stickface path and the fitted plane, but with particular attention paid to how well the plane matched the orientation of the stickface path in the crucial phase leading up to impact. At a cutoff of $0.25 \%$ of the pathlength, the average absolute residual between the stickface and the plane in the planar region was less than $5 \mathrm{~mm}$. However it can be seen from Figure 6 that, especially in the looped swings, the starting point of this region was close to where the stickface path began to move systematically away from the fitted plane. Relaxing the criterion from $0.25 \%$ to $0.50 \%$ moved the starting point of the planar region an average of $0.31 \mathrm{~m}$ further back into the downswing. The average absolute residual between the stickface and the plane remained less than 10 mm with the inclusion of the extra points, but in the looped swings the systematic deviation seen in these points caused the orientation of the fitted plane to start to move away from that in which the stickface was moving in the crucial late downswing. This divergence became more pronounced as the criterion was relaxed further. As a result it was decided that $0.25 \%$ of the path length would be used as the cutoff value in this study.

It should also be noted that the planar section of Shin et al. (2008) and the impact portion of Kwon and Casebolt (2009) both include the discontinuity that will result from impact between the ball and the club. The ball velocity after impact depends on the clubhead's velocity at impact, and the motion up to this instant is most relevant to the outcome of the shot. During the early follow-through the clubhead may continue to move close to the plane of motion of the approach to impact, but we considered it most appropriate to use only pre-impact points to calculate the swing plane. 
The stickface swing plane in field hockey is inclined at a shallower angle to the horizontal than the clubhead plane in golf. The inclination for the field hockey hit averaged $40.5^{\circ}$ across all 13 participants, and was as low as $24.5^{\circ}$ in Participant L6. Shin et al. (2008) reported that the inclination of the swing plane to the horizontal in golf varied from $49.8^{\circ}$ for a driver to $63.1^{\circ}$ for a pitching wedge. The swing plane in golf was shallower for longer clubs (Shin et al., 2008), but the even shallower swing plane in field hockey is not due to a longer stick: The sticks in this study averaged $0.93 \mathrm{~m}$ in length, which is comparable to the shortest of the three golf clubs (the pitching wedge).

\section{Conclusion}

A new method has allowed the planarity of the field hockey hit to be investigated with greater precision than has been possible in previous studies of golf. Analysis of the swings of experienced female players has established that the stickface motion is planar during much of the downswing of the field hockey hit, and for the period when most of the speed is added to the stickface. Thus the assumption of planarity would seem to be reasonable for modeling the motion of the stickface. Further investigation is needed into whether the stick shaft and the arms can also be modeled as moving in the same plane as the stickface. 


\section{References}

Abdel-Aziz, Y.L. \& Karara, H.M. (1971). Direct linear transformation from comparator coordinates into object coordinates in close-range photogrammetry. In Proceedings of the ASP Symposium on Close-Range Photogrammetry (pp. 118). Falls Church, VA: American Society of Photogrammetry.

Brétigny, P., Seifert, L., Leroy, D. \& Chollet, D. (2008). Upper limb kinematics and coordination of short grip and classic drives in field hockey. Journal of Applied Biomechanics, 24, 215-223.

Chivers, L. \& Elliott, B.C. (1987). The penalty corner in field hockey. Excel, 4, 5-8.

Cochran, A. \& Stobbs, J. (1968). The search for the perfect swing. Philadelphia: J.B. Lippincott.

Coleman, S.G.S. \& Anderson, D. (2007). An examination of the planar nature of the golf club motion in the swings of experienced players. Journal of Sports Sciences, 25, 739-748.

Elliott, B.C. \& Chivers, L. (1988). A three dimensional cinematographic analysis of the penalty corner hit in field hockey. In G. de Groot, A.P. Hollander, P.A. Huijing \& G.J. van Ingen Schenau (Eds.) Biomechanics XI-B (pp. 791-797). Amsterdam: Free University Press.

Gordon, B.J. \& Dapena, J. (2006). Contributions of joint rotations to racquet speed in the tennis serve. Journal of Sports Sciences, 24, 31-49.

Grabow, B.S., Boyle, J.M., Dongarra, J.J. \& Moler, C.B, (1977). Matrix eigensystem routines - EISPACK guide extension. Berlin: Springer-Verlag.

Jorgensen, T.P. (1999). The physics of golf (2nd ed.). New York: Springer-Verlag.

Kwon, Y.-H. \& Casebolt, J. (2009). Kinematic analysis of sports movements: golf swing plane analysis. In A.J. Harrison, R. Anderson \& I. Kenny (Eds.), 
Proceedings of the XXVIIth International Conference on Biomechanics in Sports. Retrieved November 6, 2010, from http://w4.ub.uni-konstanz.de/cpa/article/view/3041/2848

Nievergelt, Y. (1994). Total Least Squares: state-of-the-art regression in numerical analysis. SIAM Review, 36, 258-264.

Pourcelot, P., Audigié, F., Degueurce, C., Denoix, J.M. \& Geiger, D. (1997). A numerical method to synchronise home video cameras using the D.L.T. method. Medical and Biological Engineering and Computing, 35 (suppl., Part 2), 715.

Pourcelot, P., Audigié, F., Degueurce, C., Geiger, D. \& Denoix, J.M. (2000). A method to synchronise cameras using the direct linear transformation technique. Journal of Biomechanics, 33, 1751-1754.

Shakarji, C.M. (1998). Least-squares fitting algorithms of the NIST Algorithm Testing System. Journal of Research of the National Institute of Standards and Technology, 103, 633-641.

Shin, S., Casebolt, J., Lambert, C., Kim, J.-W. \& Kwon, Y.-H. (2008). A 3-D determination and analysis of the swing plane in golf. In Y.-H. Kwon, J. Shim, J.K. Shim \& L-S. Shin (Eds.), Proceedings of the XXVIth International Conference on Biomechanics in Sports. Retrieved November 6, 2010, from http://w4.ub.uni-konstanz.de/cpa/article/view/1927/1795

Sprigings, E.J. \& MacKenzie, S.J. (2002). Examining the delayed release in the golf swing using computer simulation. Sports Engineering, 5, 23-32.

Walton, J.S. (1981). A biomechanical investigation of human movement during the golf swing. Unpublished doctoral dissertation, Pennsylvania State University, University Park, PA. 
Wein, H. (1979). The science of hockey (2nd ed.) (D. Belchamber, Trans.). London: Pelham Books. (Original work published 1968).

Woltring, H.J. (1986). A FORTRAN package for generalized, cross-validatory spline smoothing and differentiation. Advances in Engineering Software, 8, 104-113.

Yeadon, M.R. \& King, M.A. (1998). A method for synchronizing digitized video data. Journal of Biomechanics, 32, 983-986. 


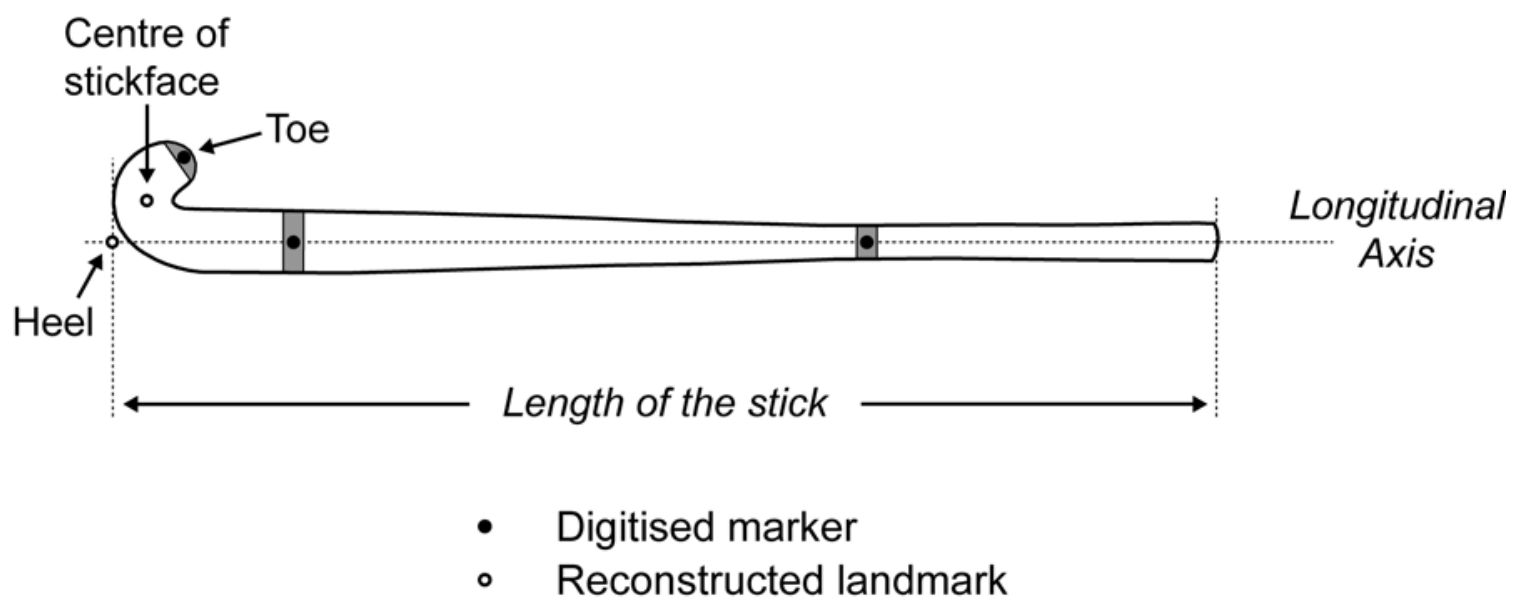

Figure 1. Locations for the three tape markers attached to the stick (shown shaded), and for the additional points whose locations were calculated from the digitised markers. 

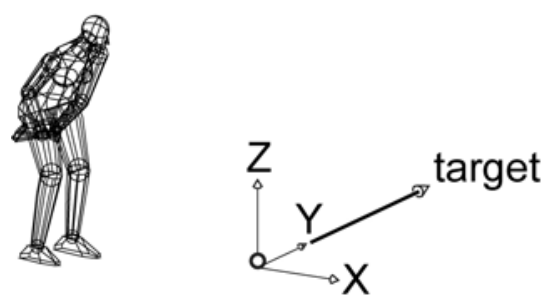

A

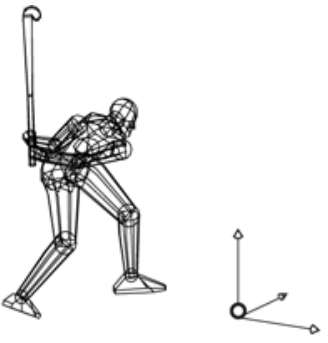

B

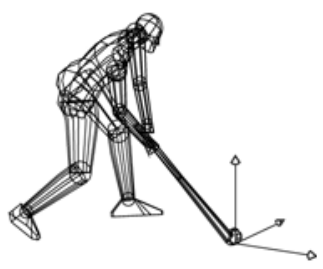

C

Figure 2. An example of the single-step hit used in this study, and the position and orientation of the XYZ reference frame. Sample body and stick positions are shown at (A) the start of the forward step with the left leg, (B) an instant near the transition between the backswing and downswing, and (C) impact. 

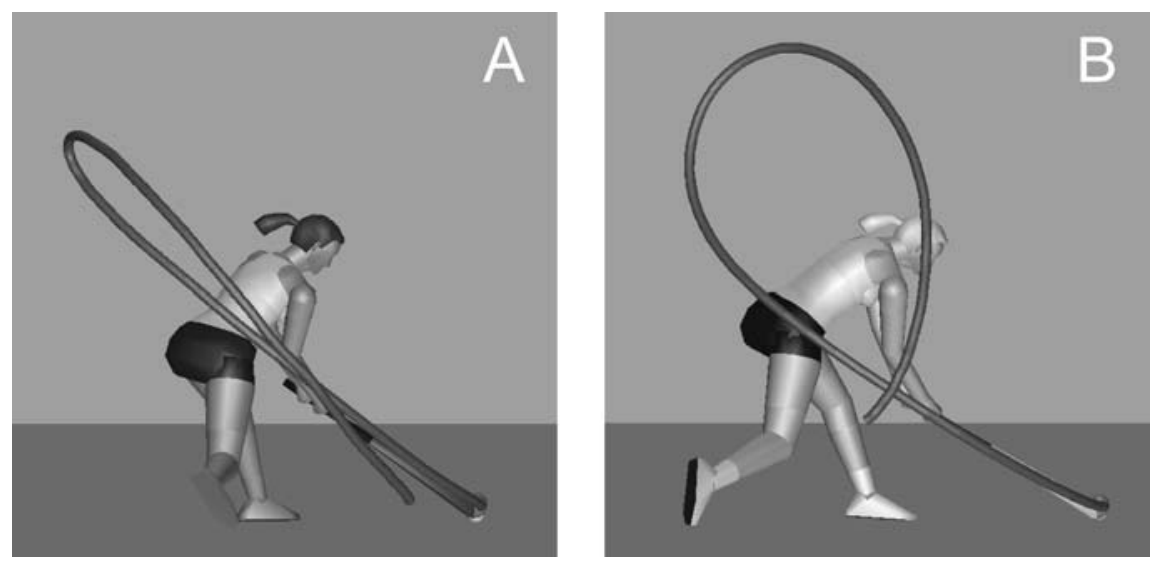

Figure 3. The path of the centre of the stickface during the entire swing for an example of (A) a straight swing (Participant S6) and (B) a looped swing (Participant $\mathrm{L1)}$. The view is from the negative $\mathrm{Y}$ axis, with the direction of the hit into the page. The body is shown at the instant of impact. 


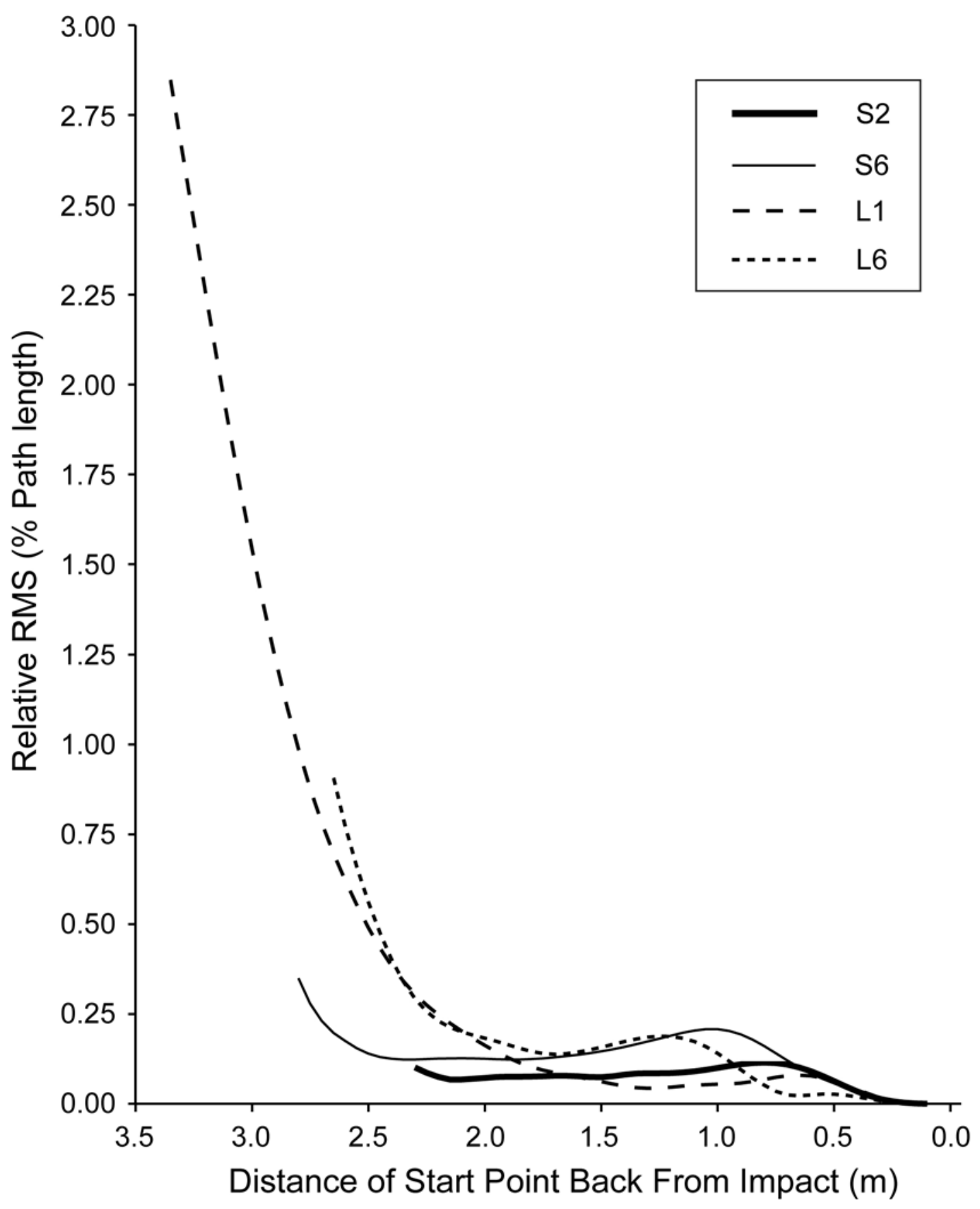

Figure 4. The degree of fit, as measured by relative RMS, between the stickface coordinates and the best-fit plane for different sections of the downswing. All sections ended at the impact position. The variation in fit is shown for two selected participants who used straight swings (S2 and S6), and two who used looped swings (L1 and L6). 

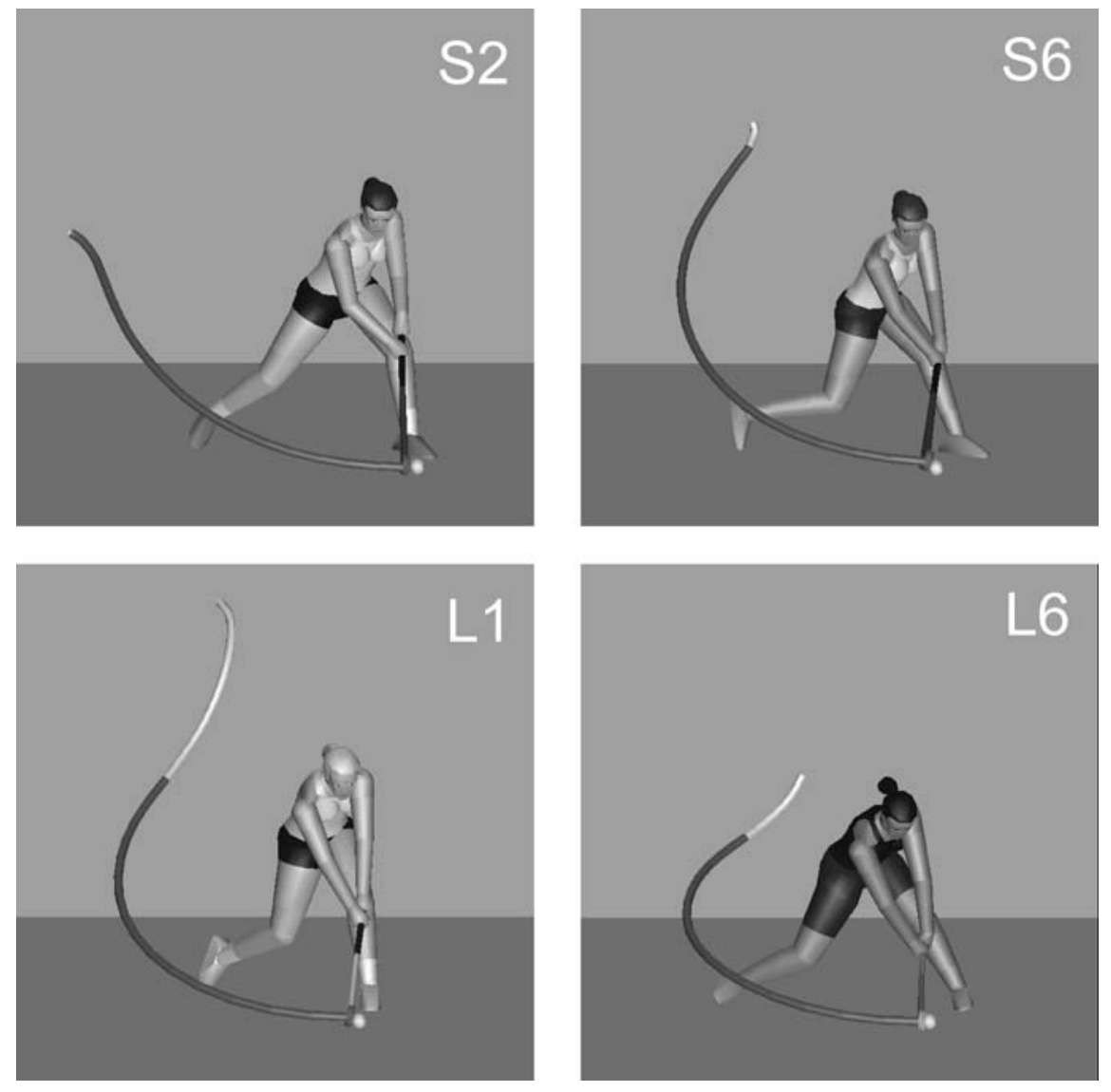

Figure 5. The planar section of the downswing for selected participants, based on a cutoff RRMS of $0.25 \%$ of the path length. The stickface path is shown for the entire downswing, with the planar portion shaded. 

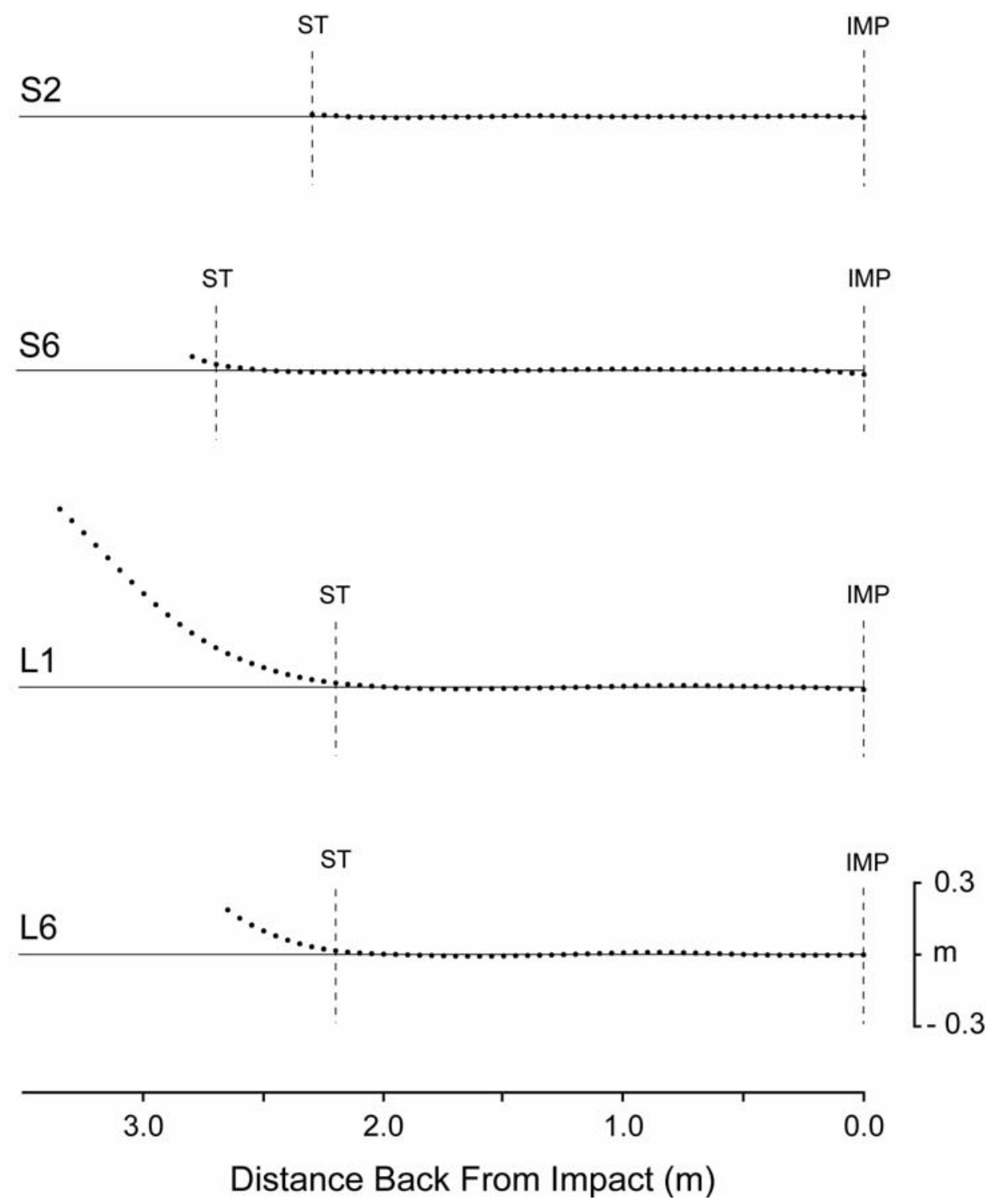

Figure 6 . The residuals between the stickface centre and the fitted plane for selected participants when an RRMS of $0.25 \%$ of the path length was chosen as the cutoff for planarity. The residuals are shown as a function of the stickface position relative to impact, and positive values indicate that the stickface was above the plane. ST = the start of the planar section when this cutoff criterion was used; IMP = impact. 